EP-30

\title{
Human hepatic alveolar echinococcosis - medical management of un resectable disease in a resource restrained setting
}

\author{
Ajaz WANI ${ }^{*}$, Dr Sadaf ALI, Omar J. SHAH \\ Department of Surgical Gastroenterology, Sher I Kashmir Institute of Medical Sciences, India
}

Introduction: Echinococcosis/hydatidosis or hydatid disease is a major human and veterinary cancer. Four species of the genus Echinococcus are known to be pathogenic to humans. Echinococcus granulosus causing cystic hydatid disease, Echinococcus multilocularis causing alveolar hydatid disease, Echinococcus vogeli and Echinococus Oligarthus cause a disease with a Polycystic pattern which affects various organs. Alveolar echinococcosis (AE) is amongst the most dangerous zoonoses known. Transmission of AE to humans is by consumption of parasite eggs excreted with faeces of definitive hosts. The disease has a long latent period and a fatal outcome if not treated timely and aggressively. Liver is the most common organ involved. This disease is now increasingly being reported from previously unaffected areas. Associated morbidity, treatment related costs and DALY's (disease adjusted life years) are high.

Methods: Complete surgical excision is the treatment of choice. Management of un resectable disease is not clear. Facilities for complex resections and liver transplant are not routinely available in developing countries. Role of pharmacotherapy in the management of AE is not well defined. We report our experience of treating 4 such patients of hepatic AE with combination chemotherapy with Albendazole and Praziquantel.

Results: Four patients were treated with Albendazole $400 \mathrm{mg}$ twice daily and Praziquantel $600 \mathrm{mg}$ on alternate days. Mean follow-up was for 2 years. The liver lesions demonstrated stability and no adverse events were related to drug use.

Conclusions: Combination chemotherapy with Albenzdazole and Praziquantel is safe and can be used for patients with un resectable disease. 